Recepción: 26/ 04/ 2017

Aceptación: 29/ 05/ 2017

Publicación: 01/ 09/ 2017

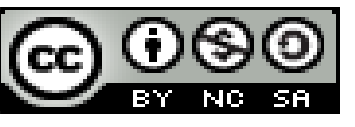

Ciencias sociales y políticas

Artículo original

\title{
Intervención del Trabajo Social en el programa Joaquín Gallegos Lara- Ecuador
}

Intervention of Social Work in the program Joaquin Gallegos Lara-Ecuador

Intervenção do Serviço Social no programa Joaquin Gallegos Lara-Ecuador

\author{
Ligia G. Parraga-Vélez ${ }^{\mathrm{I}}$ \\ ligiageoconda15@hotmail.com \\ Marjorie M. Gómez-Zambrano "I \\ marjorie.gomez@uleam.edu.ec
}

Correspondencia: ligiageoconda15@hotmail.com

I Magister en Gestión y Desarrollo Social, Licenciada en Trabajo Social, Docente de la Universidad Laica Eloy Alfaro de Manabí, Manta, Ecuador.

II Magister en Gerencia Educativa, Diploma Superior en Mediación, Licenciada en Trabajo Social, Docente de la Universidad Laica Eloy Alfaro de Manabí, Manta, Ecuador. 


\section{Resumen}

La ponencia que se presenta, tuvo como objetivo fundamental evaluar la intervención del Trabajo Social en el programa Joaquín Gallegos Lara-Ecuador. Dicho programa, auspiciado en el año 2009 por la Vicepresidencia de la República del Ecuador, emprende la Misión Solidaria Manuela Espejo para aplicar un estudio bio psicosocial y genético de los diferentes tipos de discapacidades que existen en la población ecuatoriana, con la finalidad de asignar el beneficio a quienes lo requieran, dando lugar al Programa Joaquín Gallegos Lara, dirigido a las personas con discapacidad que viven en extrema pobreza, recibiendo un bono de 240 dólares. Para ello se utilizó una metodología de corte descriptivo, de campo y documental a través de la técnica de la encuesta y visitas programadas en la que se utilizaron fichas para asentar la información y entrevistas estructuradas. Entre sus resultados se pudo obtener un diagnóstico de las situaciones más vulnerables que requieren ser atendidas con prioridad.

Palabras clave: Trabajo social; discapacidad; Joaquín Gallegos Lara; Misión Manuela Espejo.

\section{Abstract}

The main objective of the presentation was to evaluate the intervention of Social Work in the Joaquín Gallegos Lara-Ecuador program. This program, sponsored in 2009 by the Vice Presidency of the Republic of Ecuador, undertakes the Manuela Espejo Solidarity Mission to apply a biopsychosocial and genetic study of the different types of disabilities that exist in the Ecuadorian population, in order to allocate the benefit to those who require it, giving rise to the Joaquín Gallegos Lara Program, aimed at people with disabilities living in extreme poverty, receiving a bonus of 240 dollars. For this purpose, a descriptive, field and documentary method was used through the technique of the survey and scheduled visits in which cards were used to record the information and structured interviews. Among its results, it was possible to obtain a diagnosis of the most vulnerable situations that require priority attention.

Keywords: Social work; disability; Joaquín Gallegos Lara; Mission Manuela Espejo.

\section{Resumo}

O objetivo principal da apresentação foi avaliar a intervenção do Serviço Social no programa Joaquín Gallegos Lara-Ecuador. O programa, patrocinado em 2009 pelo Vice-Presidente da 
República do Equador, compromete-se a Missão Manuela Espejo Solidariedade para implementar um estudo bio psicossocial e genética de diferentes tipos de deficiências que existem na população equatoriana, a fim de atribuir o benefício para aqueles que o exigem, dando origem ao Programa Joaquín Gallegos Lara, destinado a pessoas com deficiência que vivem em extrema pobreza, recebendo um bônus de 240 dólares. Para tanto, utilizou-se um método descritivo, de campo e documental, por meio da técnica da pesquisa e das visitas agendadas em que os cartões foram utilizados para registrar as informações e as entrevistas estruturadas. Entre seus resultados, foi possível obter um diagnóstico das situações mais vulneráveis que requerem atenção prioritária.

Palavras chave: Trabalho social; incapacidade; Joaquín Gallegos Lara; Missão Manuela Espejo.

\section{Introducción}

La intervención del Trabajador Social en el Programa Joaquín Gallegos Lara, juega un papel importante para la sociedad, porque es la persona encargada de realizar el estudio socio económico de las familias que cuidan a las personas con discapacidad grave severa, tanto física como intelectual con un porcentaje mayor a $75 \%$ y poseen muchas limitaciones para desenvolverse en el entorno.

Para ello, en el año 2009, la Vicepresidencia de la República del Ecuador emprende la Misión Solidaria Manuela Espejo para aplicar a un estudio bio psicosocial y genético de los diferentes tipos de discapacidades que existen, con el objetivo de obtener un diagnóstico de las situaciones más vulnerables que requieren ser atendidas con prioridad, se identifican la necesidad de ayudas técnicas como sillas de ruedas, colchón anti escaras, coche postural, en algunos casos le dotan de vivienda, cuando no cuentan con la misma o están en pésimas condiciones, así mismo con esta información poder trabajar con la población en prevención de discapacidades.

De allí, nace el Programa Joaquín Gallegos Lara dirigido a las personas con discapacidad que viven en extrema pobreza, que consiste en otorgar un bono de 240 dólares a un familiar cuidador o quien esté como responsable del cuidado de una persona que padece discapacidad física muy grave o intelectual severa que no pueden realizar las actividades de la vida diaria, teniendo a la vez que cumplir con requisitos para adquirir el beneficio, dinero que debe ser invertido en las necesidades como la medicina, pañales, rehabilitación, nutrientes y otras que deben ser 
justificadas con facturas para dar a conocer que el dinero es utilizado para la persona con discapacidad, de lo contrario a través de seguimiento, puede ser retirado sin opción de volver a recibirlo.

Para que el gobierno a través del programa otorgue el bono de 240 dólares, interviene un equipo interdisciplinario: el médico con la valoración de la discapacidad, en algunos casos el aporte del psicólogo y la función del Trabajo Social que realiza un estudio minucioso para emitir un informe con resultados obtenidos a través de la aplicación de fichas, donde refleja el nivel socioeconómico, se valora también a través de la observación si la persona con discapacidad está siendo bien atendida en todos los aspectos ya que esto es fundamental para que pueda acceder al beneficio.

Una vez obtenidos los resultados, el trabajador social hace otra visita a las personas que salen favorecidas del bono para orientarle en los requisitos y responsabilidades que debe cumplir, se les llena un formulario donde consta la firma del cuidador/a de la persona con discapacidad, pero aquí no culmina todo el proceso porque una vez que empiezan a recibir el dinero se hace seguimiento para saber si existe una buena inversión en las necesidades, se solicita facturas como fuentes de verificación.

Cabe recalcar que, así como se otorga el beneficio también se les retira si se le comprueba incumplimiento con los requisitos establecidos, dado que existen cuidadores que el dinero lo gastan en ellos y dejan de atender a la persona con discapacidad.

\section{Desarrollo}

Más de mil millones de personas viven en todo el mundo con alguna forma de discapacidad; de ellas, casi 200 millones experimentan dificultades considerables en su funcionamiento. En los años futuros, la discapacidad será un motivo de preocupación aún mayor, pues su prevalencia está aumentando, sea por generarse genéticamente, por enfermedades catastróficas u otras, así como accidentes inesperados ya que ninguna persona está exenta de tener una discapacidad.

En consecuencia, la Organización Mundial de la Salud (1948) y el Grupo del Banco Mundial, han producido conjuntamente este informe mundial sobre la discapacidad para proporcionar datos destinados a la formulación de políticas y programas innovadores que mejoren las vidas de las 
personas con discapacidades y faciliten la aplicación de la Convención de Naciones Unidas sobre los Derechos de las Personas con Discapacidad, que entró en vigor en mayo de 2008.

En el mundo, no todas las personas con discapacidad viven en buenas condiciones, unas por falta de atención, otras con necesidades básicas por el nivel de pobreza en que viven, limitándose muchas veces al acceso a la salud, la educación, y los servicios de transporte por falta de recursos económicos.

Por su parte, en el Artículo 6 de la Ley de Discapacidades Ecuador (CONADIS, 2017) estipula que para los efectos de esta Ley se considera persona con discapacidad a toda aquella que, como consecuencia de una o más deficiencias físicas, mentales, intelectuales o sensoriales, con independencia de la causa que la hubiera originado, ve restringida permanentemente su capacidad biológica, psicológica y asociativa para ejercer una o más actividades esenciales de la vida diaria, en la proporción que establezca el reglamento. Los beneficios tributarios previstos en esta ley, únicamente se aplicarán para aquellos cuya discapacidad sea igual o superior a la determinada. El Reglamento a la Ley podrá establecer beneficios proporcionales al carácter tributario, según los grados de discapacidad, con excepción de los beneficios establecidos en el Artículo 74.

La misma ley, en su Artículo 7, expresa que toda persona con deficiencia es considerada aquella que, presente disminución o supresión temporal de alguna de sus capacidades físicas, sensoriales o intelectuales manifestándose en ausencias, anomalías, defectos, pérdidas o dificultades para percibir, desplazarse, oír y/o ver, comunicarse, o integrarse a las actividades esenciales de la vida diaria limitando el desempeño de sus capacidades; y, en consecuencia, el goce y ejercicio pleno de sus derechos.

En estos años se han logrado considerables progresos, a pesar que aún queda por hacer. Las personas con discapacidad tienen el derecho de vivir en su comunidad, disfrutar de salud y bienestar, recibir una buena educación y tener la oportunidad de trabajar. (UNICEF, Inclusión Social, discapacidad y políticas públicas, 2005)

Básicamente, la historia de las personas con discapacidad es una historia de exclusión, en muchas sociedades, fueron rechazadas debido a temores o ignorancia, viviendo en extrema pobreza, maltratadas por sus propios familiares y vistas como un objeto. 
La inclusión tiene que ver con la respuesta apropiada a todos los aspectos de la diversidad dentro de la educación, salud o disposiciones sociales que son un elemento importante. (UNICEF, Inclusión Social, discapacidad y políticas públicas, 2005)

Existen diferentes tipos de discapacidades que se detallan a continuación:

Discapacidad auditiva sordera. - Incapacidad de oír, esta tan limitada que no puede utilizarla para las actividades de la vida diaria, aunque disponga de un aparato que amplifique los sonidos (auxiliar y auditivo). Se dice que una persona es sorda cuando su deficiencia auditiva es total o profunda, hipoacúsica si su pérdida de la audición es parcial y su audición puede mejorar con el uso de dispositivos electrónicos como los audífonos.

Normalmente son tres las causas de la discapacidad auditiva: la primera y más común es la genética, en segundo lugar, está la adquirida (es decir, que se adquiere en algún momento de la vida, como luego de un accidente) y por último están las congénitas, que son las prenatales, perinatales.

Discapacidad visual ceguera. - Incapacidad de ver, esta tan limitada para las actividades de la vida diaria. El National Eye Institute (1968), define baja visión como una deficiencia visual no corregible por estándar anteojos, lentes de contacto, medicamentos o cirugía que interfiere con la capacidad para realizar actividades cotidianas.

De acuerdo al grado de limitación de la visión, se suele distinguir entre personas ciegas, que no obtienen información a través del canal visual; y personas con disminución visual, quienes en cambio sí la adquieren mediante dicho canal. A su vez, dentro del grupo de personas con disminución visual hay diversas manifestaciones, las cuales se rigen de acuerdo al tipo de limitación, ya sea por agudeza o por campo visual. Esta clasificación no es exhaustiva, pero sirve a grandes rasgos para trazar una distinción entre los tipos de discapacidad visual más frecuentes.

Discapacidad física. - Afección de la integridad física o funcional del cuerpo de la persona y su movilidad o independencia debido a una enfermedad que se presentó antes o después del nacimiento, se da por diferentes causas como parálisis cerebral infantil, paraplejia, hemiplejia y otras. De los tipos de discapacidad que existen esta se caracteriza por la disminución parcial o total de la movilidad de uno o más miembros de tu cuerpo, lo que se traduce en una dificultad o 
impedimento a la hora de realizar diversas tareas motoras, en especial las de la motricidad fina, así como movimientos incontrolados, temblores, dificultad de coordinación, fuerza reducida, entre otros.

Discapacidad Mental e Intelectual. - Una persona con deficiencia mental es aquella que desde su infancia no aprende rápido ni recuerda cosas como otros de su edad. El nivel de afectación puede ser leve, moderado y severo o profundo. (CONADIS, (Consejo Nacional de Discapacidades), s.f.). Esta discapacidad no puede llamarse enfermedad ya que no se cura y acompañará al individuo durante toda su vida.

En este tipo de discapacidad, se destaca como la más común la hereditaria, como el síndrome de Down, aunque también existen causas congénitas o adquiridas durante la gestación (ingesta de diferentes drogas, contracción de rubéola o VIH) y por último, las adquiridas, que se producen por daños al sistema nervioso central o al cerebro (meningitis, aspiración de toxinas, etc)

Entre los tipos de discapacidad expuestos, se hace énfasis en quienes pueden ser beneficiarios del Bono Joaquín Gallegos Lara, entre ellas las que padecen de limitaciones severas como parálisis cerebral, intelectual grave, paraplejia y otras que les impide realizar las actividades de la vida diaria. Al respecto, cabe destacar que la inclusión social es un imperativo moral de la humanidad, tal como ha sido reconocido en la Declaración Universal de los Derechos Humanos, la cual consagra la igualdad de derechos y libertades, de modo que, sin distinción alguna por motivos de raza, sexo, religión, origen nacional o social o cualquier otra condición todos podamos participar en comunidades que favorezcan el desarrollo y bienestar de las personas, gozando de los derechos sin discriminación alguna. (Jaramillo, 2014).

\section{Metodología}

La metodología fue de tipo descriptiva, documental y de campo. Se describieron la información socioeconómica de las personas con discapacidad y sus familias posteriores a las visitas programadas, para ello se utilizaron fichas para asentar la información. Asimismo, se realizaron entrevistas estructuradas a familiares que permitieron determinar la asignación de las ayudas técnicas por medio de la Misión Solidaria Manuela Espejo, identificando a las familias que no habían adquirido ningún tipo de beneficio. 
También, se hizo el análisis de la información obtenida como producto de la ficha y la entrevista,

y se les otorgo a las personas el bono Joaquín Gallegos a todas aquellas familias que cumplían con lo exigido por el programa. Es de señalar que en esta evaluación intervino un equipo interdisciplinario como es Trabajador/a Social, Psicólogo/a y Médico/a, quienes a través de un informe dieron a conocer los casos más relevantes para que obtengan los beneficios, no se dejó a un lado el uso de la técnica de observación que permitió identificar el entorno en el que se desenvuelve la persona con discapacidad, el trato y cuidado que reciben del familiar o de quienes se encuentran como cuidadores.

\section{Conclusiones}

En las visitas domiciliarias se identificó a los beneficiarios de acuerdo al estudio socioeconómico con la intervención del Trabajo Social, así mismo brindó orientación a las familias cuidadoras en los requisitos para que actualizaran el carnet de discapacidad.

A las personas beneficiarias se les aplicó el formulario con los datos requeridos y firma de cuidador/a para la atención de las personas con discapacidad.

Se emitieron los informes del trabajo social realizado a las personas beneficiarias y se realizan el seguimiento para conocer si están haciendo buen uso del dinero que reciben, en cuanto a control médico, rehabilitación, transporte, vestimenta, ambiente agradable, cuidado en el hábito de la vida diaria y alimentación adecuada.

Se logró la valoración médica, psicológica y social de la población con Discapacidad en el Ecuador por parte de un equipo multidisciplinario constituido por un equipo multidisciplinario que incluían Trabajador/a Social, Psicólogo/a y Médico/a.

En el Programa Joaquín Gallegos Lara se logró atender aproximadamente a 14.479 beneficiarios con el bono de 240 dólares y ayudas técnicas como sillas de ruedas, coche postural, pañales.

\section{Referencias Bibliográficas}

CONADIS (s/f). Consejo Nacional de Discapacidades del Ecuador. 
Convención de Naciones Unidas sobre los Derechos de las Personas con Discapacidad (2008). https://web.archive.org/web/20080827181725/http://www.disabilityworld.org/01-

03_03/spanish/noticias/unconvention.shtml

Jaramillo, J (2014). Estrategias para la inclusión de niños, niñas y adolescentes en contextos

familiares. https://dialnet.unirioja.es/descarga/articulo/4798450.pdf.

OMS (1948). Organización Mundial de la Salud.

NEY (1968). National Eye Institute. United States Public Health Service. Bethesda, Maryland, Estados Unidos

UNICEF (2005). Inclusión Social, discapacidad y políticas públicas. 